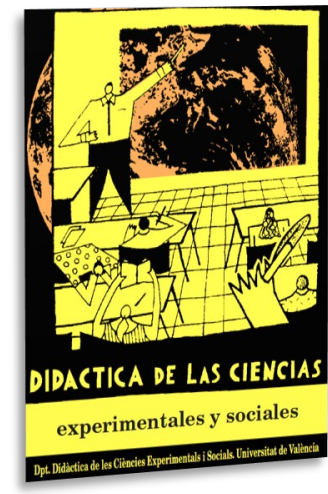

\title{
Trabajos Prácticos de Laboratorio y Modelos didácticos: una propuesta de clasificación
}

\author{
Practical Laboratory Work and Didactic \\ Models: a classification proposal
}

DOI: $10.7203 / D C E S .40 .18056$

\author{
Erica Gabriela Zorrilla \\ Consejo Nacional de Investigaciones Cientificas y Técnicas (CONICET)- Universidad Nacional de \\ San Juan (IIECE, FFHA-UNSJ) (Argentina), ezorrilla@ffha.unsj.edu.ar \\ ORCID iD: http://orcid.org/0000-0001-6460-3319
}

\author{
Claudia Alejandra Mazzitelli \\ Consejo Nacional de Investigaciones Cientificas y Técnicas (CONICET) - Universidad Nacional de \\ San Juan (IIECE, FFHA-UNSJ) (Argentina), mazzitel@ffha.unsj.edu.ar \\ ORCID iD: http://orcid.org/0000-0002-1199-4843
}

\begin{abstract}
Resumen: Los Trabajos Prácticos de Laboratorio no siempre contribuyen a la construcción de los conocimientos de los estudiantes, tal como lo señalan muchos autores. Atendiendo a que esto puede atribuirse a distintos aspectos, es necesario analizarlos y clasificarlos teniendo en cuenta no solo las características del trabajo experimental, sino también considerando que las diferencias en sus aportes podrían encontrarse fundamentadas en el modelo didáctico que subyace a la práctica docente. En este artículo se presenta una propuesta de clasificación en la que se integran elementos de las clasificaciones más utilizadas tanto para los Trabajos Prácticos de Laboratorio como para los modelos didácticos. Esta clasificación pretende ser una herramienta que pueda contribuir con el análisis de las prácticas experimentales y la explicitación de los supuestos de base, reflexionando sobre su alcance tanto en la enseñanza como en el aprendizaje.
\end{abstract}

Palabras Clave: trabajos prácticos de laboratorio, modelos didácticos, clasificación, Ciencias Naturales, didáctica de las Ciencias Experimentales

ABSTRACT: Practical Laboratory Work does not always contribute to the construction of student's knowledge, as many authors point out. Given that this can be attributed to different aspects, it is necessary to analyze and classify them taking into account not only the characteristics of the experimental work, but also considering that the differences in their contributions could be based on the didactic model that underlies the teaching practice. This article presents a classification proposal in which elements of the most used classifications are integrated, both for the Practical Laboratory Work and for the didactic models. This classification aims to be a tool that can contribute to the analysis of experimental practices and the explanation of the basic assumptions, reflecting on their scope in both teaching and learning.

KEYWORDS: practical laboratory work, didactic models, classification, Natural Sciences, Experimental Sciences teaching

Fecha de recepción: julio de 2020

Fecha de aceptación: abril de 2021 


\section{INTRODUCCIÓN}

La enseñanza de las Ciencias Naturales se ha desarrollado tradicionalmente de manera teóricopráctica (Chinchilla Buelvas, 2017), atendiendo a que una de las características de las Ciencias Naturales es la de ser experimental, y es por esto que las actividades de laboratorio ocupan un lugar destacado en las propuestas de aula. Múltiples investigaciones realizadas en esta área (Bastida de la Calle, Ramos Fernández y Soto López, 1990; Hodson, 1994; Millar, 2009; Fernández, 2013; Valencia, 2017), evidencian un amplio consenso sobre la importancia de las actividades de laboratorio para la educación en Ciencias Naturales, ya que proporcionan la oportunidad de que los y las estudiantes desarrollen distintas competencias científicas básicas como el pensamiento crítico y habilidades vinculadas a la observación, construcción de hipótesis y análisis de resultados. No obstante, a veces, el Trabajo Práctico de Laboratorio (TPL) resulta poco útil desde el punto de vista pedagógico, ya que, en ocasiones, su implementación en las aulas presenta diferentes obstáculos (Valencia y Torres, 2017) lo cual deriva en la reproducción acrítica de experiencias tipo receta (Idoyaga y Maeyoshimoto, 2018) y por esto no contribuyen al logro de los objetivos de aprendizaje propuestos.

Reconociendo que los TPL pueden o no contribuir a la construcción de los conocimientos de los estudiantes, dependiendo de las actividades que se propongan, surge la necesidad de analizar los distintos tipos de TPL. Flores, Caballero Sahelices y Moreira (2009) señalan que es necesario avanzar en el estudio de los TPL desde una visión más general e integral de enseñanza y de aprendizaje en el laboratorio de Ciencias Naturales. De esta manera, las diferencias de los aportes de los TPL podrían encontrarse fundamentadas en el modelo didáctico que subyace a la práctica docente (Zorrilla, 2019), por lo que resulta relevante proponer una clasificación de los TPL teniendo en cuenta no sólo las características del trabajo experimental, sino también los modelos didácticos que los sustentan.

Por lo expuesto, en este trabajo se presentan algunas de las clasificaciones más utilizadas para los TPL y para los modelos didácticos empleados en la enseñanza de las Ciencias Naturales, así como una propuesta de clasificación en la que se integran ambos aspectos, con el fin de contribuir con el análisis de las prácticas experimentales.

\section{ACERCA DEL CONCEPTO DE TRABAJO PRÁCTICO DE LABORATORIO}

El término Trabajos Prácticos se utiliza con frecuencia para referirse a las actividades de enseñanza de las Ciencias Naturales, en las que los alumnos han de utilizar determinados procedimientos para resolver las situaciones que se plantean (Fernández, 2013). Los Trabajos Prácticos (TP) suponen la articulación de diferentes tipos de actividades, mediante un enfoque integrado, en el que la teoría y la práctica se entrelazan en un tratamiento conjunto (del Carmen, 2011). Sin embargo, no todos los TP se llevan a cabo en un laboratorio, de esta manera, se puede reconocer a los TPL como un subconjunto de una categoría más general, los TP.

A pesar de que los TPL son un tema ampliamente trabajado por docentes e investigadores en enseñanza de las Ciencias Naturales, son pocas las referencias que definen qué es concretamente un TPL. Esto se evidencia en la misma práctica áulica, porque inclusive cuando los docentes son los encargados de definir qué es un TPL, lo hacen brindando una serie de características y no una definición acabada de los mismos (Antúnez, Pérez y Petrucci, 2008).

Al respecto, Hodson (1994) señala que no todos los TPL son experimentos. Continuando con esa idea, Leite y Figueroa (2004) diferencian entre trabajo práctico -realizado por el alumnado manipulando recursos y materiales diversos, no necesariamente experimental-, trabajo laboratorial trabajo práctico realizado en el laboratorio o con equipamientos específicos de laboratorio-, y trabajo experimental -trabajo práctico que implica la manipulación de variables, sea en forma de una experiencia guiada o como investigación-. 
Por otra parte, Richoux y Beaufils (2003) indican que los TPL consisten en poner a disposición de los estudiantes una ficha de actividades y aparatos adecuados para estudiar fenómenos, generalmente de forma cuantitativa. Además, otros autores (Tamir y García Rovira, 1992; Barberá y Valdés, 1996; del Carmen, 2000, 2011 y Fernández, 2013), acuerdan en plantear que los TPL son actividades realizadas por los alumnos, aunque con un grado variable de participación en su diseño y ejecución, presentando las siguientes características distintivas: a) implican el uso de procedimientos científicos diferentes: observación, formulación de hipótesis, realización de experimentos, técnicas manipulativas, elaboración de conclusiones, entre otros; b) requieren del uso de un material específico, semejante al utilizado por los científicos, aunque a veces simplificado para facilitar su empleo por los alumnos, y c) son actividades más complejas de organizar que las de lápiz y papel.

Así, teniendo en cuenta lo expuesto, puede decirse que los TPL deberían brindarle a los estudiantes la posibilidad de aprender a partir de sus propias experiencias, convirtiéndose en un importante recurso para el aprendizaje de muchos de los contenidos conceptuales, procedimentales y actitudinales (Merino y Herrero, 2007). También deberían ser usados para estimular la curiosidad y el placer por la investigación y el descubrimiento, otorgando a los alumnos la posibilidad de explorar, manipular, sugerir hipótesis, entre otras cuestiones (Gil, 1997; Carrascosa, Vilches y Valdés, 2006; Bravo y otros, 2016), lo que permite el desarrollo de los procedimientos propios del quehacer científico. Además, en relación con la formación docente, deben constituir un recurso didáctico que influye en el proceso de formación inicial y en el futuro desempeño docente (De Pro Bueno, 1998; De Pro Bueno, 1999; Valencia y Torres, 2017).

\section{CLASIFICACIÓN DE LOS TRABAJOS PRÁCTICOS DE LABORATORIO}

Como ya se mencionó, a los TPL se les atribuyen múltiples beneficios, presentándolos como un recurso motivador para favorecer el aprendizaje de contenidos no solamente conceptuales y procedimentales, sino también, actitudinales (Caamaño, 1992; García Ruiz y Calixto Flores, 1999; Merino y Herrero, 2007; Walz, Weisz y Albarenque, 2013), como una importante influencia en la creación de hábitos de trabajo (como la rigurosidad o el espíritu de colaboración, entre otros); y como un factor asociado a la construcción de la confianza en los estudiantes en cuanto a la confianza en la capacidad para resolver problemas. A pesar de esto, en la realidad áulica el tiempo que se les dedica a los mismos suele ser reducido, lo cual puede atribuirse a diferentes factores como el excesivo número de alumnado, la falta de instalaciones o recursos adecuados y la escasa formación docente (Fernández, 2010; Nappa, Vázquez, Maratta y Mazzitelli, 2015; Zorrilla, Mazzitelli, Calle Restrepo, Angulo Delgado y Soto Lombana, 2020).

Según Hodson (1994), gran parte de las prácticas que se realizan están mal concebidas, son confusas y carecen de valor educativo real. Esta afirmación se refiere principalmente al estilo exclusivamente demostrativo de las prácticas, el cual es el más popular, pero asimismo el más criticado. Este tipo de prácticas tienen como característica distintiva que se parecen a recetas de cocina, presentando un desarrollo completamente estructurado, en el que los estudiantes han de limitarse a seguir un guion previamente elaborado sin su participación, y en donde no se encuentran actividades que fomenten aspectos esenciales del trabajo científico, tales como el planteamiento de problemas, la emisión de hipótesis, la elaboración de posibles diseños para su contrastación, el análisis crítico de los resultados, entre otras (Jiménez Valverde, Llobera Jiménez y Llitjós Viza, 2006; Flores, Caballero Sahelices y Moreira, 2009).

Debido a la variedad de características que pueden presentar los TPL, y teniendo en cuenta que por esta razón pueden existir diferentes tipos de TPL (Hodson, 1994), resulta necesario realizar una clasificación de los mismos. Para esto, resulta útil considerar la definición de nivel de apertura como la proporción en la que el docente facilita los problemas, las maneras y medios para afrontarlos y las respuestas a los mismos (Schwab, 1962). 
Una de las propuestas de clasificación de los TPL que tiene en cuenta los posibles niveles de apertura es la presentada por Herron (1971), quien diferencia las prácticas experimentales según sus objetivos y la proporción en que están dados los materiales, métodos y soluciones. En la Tabla 1 se presenta una síntesis de su propuesta:

TABLA 1. Tipos de Trabajos Prácticos de Laboratorio según Herron

\begin{tabular}{ccccccc}
\hline Nivel & Nombre & Objetivo & Material & Método & Solución & Estilo de práctica \\
\hline 0 & Demostración & Dado & Dado & Dado & Dada & Expositivo \\
\hline 1 & Ejercicio & Dado & Dado & Dado & Abierta & Expositivo \\
\hline 2 & $\begin{array}{c}\text { Investigación } \\
\text { estructurada }\end{array}$ & Dado & $\begin{array}{c}\text { Dado todo o } \\
\text { en parte }\end{array}$ & $\begin{array}{c}\text { Dado en } \\
\text { parte o } \\
\text { abierto }\end{array}$ & Abierta & $\begin{array}{c}\text { Expositivo- } \\
\text { Investigación }\end{array}$ \\
\hline 3 & $\begin{array}{c}\text { Investigación } \\
\text { abierta }\end{array}$ & Dado & Abierto & Abierto & Abierta & Investigación \\
\hline 4 & Proyecto & $\begin{array}{c}\text { Dado en } \\
\text { parte o } \\
\text { abierto }\end{array}$ & Abierto & Abierto & Abierta & Investigación \\
\hline
\end{tabular}

Fuente: Herron, 1971

Por otra parte, Priestley (1997) propone una escala de siete niveles de apertura para clasificar las actividades prácticas de laboratorio y señala, para cada uno de los niveles, los procesos cognitivos que se favorecen. En la Tabla 2 se presenta una síntesis de esta clasificación:

TABLA 2. Tipos de Trabajos Prácticos de Laboratorio según Priestley

\begin{tabular}{|c|c|c|c|}
\hline Nivel & Título & Actividades realizadas & $\begin{array}{l}\text { Proceso cognitivo } \\
\text { requerido }\end{array}$ \\
\hline 1 & $\begin{array}{l}\text { Herméticamente } \\
\text { cerrado }\end{array}$ & $\begin{array}{l}\text { Se proporcionan todos los procedimientos. Los estudiantes } \\
\text { apuntan los datos en los espacios reservados de un informe } \\
\text { de laboratorio. Se incluyen tablas con datos }\end{array}$ & Conocimiento \\
\hline 2 & Muy cerrado & $\begin{array}{l}\text { Se proporcionan todos los procedimientos a los estudiantes. } \\
\text { Se incluyen tablas de datos }\end{array}$ & Conocimiento \\
\hline 3 & Cerrado & Se proporcionan todos los procedimientos a los estudiantes & $\begin{array}{l}\text { Conocimiento y } \\
\text { comprensión }\end{array}$ \\
\hline 4 & Entreabierto & $\begin{array}{l}\text { Se proporcionan todos los procedimientos a los estudiantes. } \\
\text { Algunas preguntas o conclusiones son abiertas }\end{array}$ & $\begin{array}{l}\text { Comprensión y } \\
\text { aplicación }\end{array}$ \\
\hline 5 & $\begin{array}{l}\text { Ligeramente } \\
\text { abierto }\end{array}$ & $\begin{array}{l}\text { Se proporcionan la mayoría de procedimientos a los } \\
\text { estudiantes y algunas preguntas o cuestiones son abiertas }\end{array}$ & Aplicación \\
\hline 6 & Abierto & $\begin{array}{l}\text { Los estudiantes desarrollan sus propios procedimientos. Se } \\
\text { les proporciona una lista con el material. Muchas preguntas } \\
\text { o conclusiones son abiertas }\end{array}$ & Análisis y síntesis \\
\hline 7 & Muy abierto & $\begin{array}{l}\text { A los estudiantes se les indica un problema que tienen que } \\
\text { resolver o que ellos mismos proponen. Luego desarrollan el } \\
\text { procedimiento y sacan sus conclusiones }\end{array}$ & $\begin{array}{l}\text { Síntesis y } \\
\text { evaluación }\end{array}$ \\
\hline
\end{tabular}

Fuente: Priestley, 1997 
De esta manera, las prácticas con mayores niveles de apertura (nivel 7 según Priestley, nivel 4 según Herron) son aquellas en las que los estudiantes deben diseñar, desarrollar y conducir su propio experimento, formular hipótesis y predecir el resultado, potenciando el uso de recursos cognitivos de alto orden $\mathrm{y}$, además, favoreciendo una mejor actitud de los estudiantes hacia la investigación científica, asociando más claramente los conceptos teóricos con los datos empíricos. En el otro extremo se encuentran las prácticas tradicionales (nivel 1 según Priestley, nivel 0 según Herron), las cuales además de ser poco representativas de la actividad científica, no permiten integrar la práctica con los conceptos y proposiciones conocidas con anterioridad, proceso que contribuye con la construcción del conocimiento.

Otra clasificación posible para los TPL, es la efectuada por Petrucci, Ure y Salomone (2011), quienes analizan dos dimensiones relativas a los TPL: el nivel de apertura y el control de variables. La primera de estas dimensiones permite clasificar a los TPL según sean abiertos o cerrados, es decir, teniendo en cuenta si el diseño y la ejecución del mismo lo realizan los alumnos o los docentes. Es una dimensión continua, ya que un TPL puede situarse en algún punto entre dos extremos. En un extremo se ubican los TPL totalmente estructurados y en el otro los completamente desestructurados. La segunda dimensión se refiere a si en los TPL se involucran mediciones o son de tipo cualitativos. Así, aquellos TPL que no involucran mediciones simplemente se los denomina como experiencias.

En las clasificaciones anteriormente expuestas, puede observarse que las prácticas cerradas que son las que mayoritariamente utilizan los docentes (Pavón et al, 2009; Insausti y Merino, 2016; Valencia y Torres, 2017; Cano, 2019; Cruz, Sanz y León, 2019)-, varían entre los niveles 0 y 2 en la propuesta de Herron, 1 a 3 en la estructura presentada por Priestley y según Petrucci, Ure y Salomone se corresponden con lo que denominan "guías receta".

De esta manera, si estos tipos de TPL, en los que el alumnado ha de limitarse a seguir una serie de pasos determinados para alcanzar una conclusión final, son los más extendidos en la enseñanza de las ciencias, entonces contribuyen a la transmisión de una imagen distorsionada y simplista de ciencia (López Rua y Tamayo Alzate, 2012). Además, dificultan un mejor aprovechamiento del potencial que, en general, tienen los TPL para el aprendizaje de las ciencias experimentales.

\section{MODELOS DIDÁCTICOS EN LA ENSEÑANZA DE LAS CIENCIAS NATURALES}

Los modelos didácticos son entendidos como esquemas mediadores entre la realidad y el pensamiento, estructuras en torno a las cuales se organiza el conocimiento que tendrá siempre un carácter provisorio y aproximado a la realidad (Guirado, 2013).

El modo de desarrollar la actividad docente y los saberes implícitos respecto a dicha actividad se encuentran relacionados, dado que en muchos casos se suele enseñar del modo como uno ha sido enseñado (Porlán, Rivero y Martín, 1997 y 1998; Martínez Aznar et al., 2001; Jiménez Llanos y Correa Piñero, 2002; Rodríguez Garrido y Meneses Villagrá, 2011). De esto se desprende la importancia de conocer lo "que piensan" los docentes y su relación con lo "que hacen", porque la interacción de estas dos variables puede dar lugar al surgimiento de dificultades en la enseñanza o en el aprendizaje. Es por esto que el concepto de modelo didáctico sostenido por los docentes, representa una importante herramienta intelectual para abordar los problemas de la enseñanza en el aula, ayudándonos a establecer el necesario vínculo entre el análisis teórico y la intervención práctica (Chrobak y Leiva Benegas, 2006)

Al estudiar los modelos didácticos para la enseñanza de las Ciencias Naturales, pueden encontrase varias clasificaciones. Es así, que se considera importante conocer los aportes de autores que permiten analizar las actividades experimentales en relación con diferentes modelos (Fernández y Elortegui, 1996, Fernández et al., 1997; Fernández, Elortegui y Medina, 2002), caracterizando cómo los docentes enseñan los contenidos científicos, teniendo en cuenta la selección y estudio de algunos aspectos asociados al pensamiento del docente y a la práctica educativa. Sus estudios proponen cinco modelos: el modelo transmisor receptor, el modelo tecnológico cientificista, el modelo artesano 
humanista, el modelo por descubrimiento investigativo y el modelo constructivista reflexivo. Estos autores destacan, además, que cualquier práctica de enseñanza que realicen los docentes responde a una teoría específica, pero esto no implica que dicha relación sea explícita, sino todo lo contrario, en la mayoría de los casos se encuentra implícita.

Ruiz Ortega (2007) realiza otro aporte a la clasificación de los modelos didácticos específicos de la enseñanza de las ciencias. Este autor presenta aspectos teóricos teniendo en cuenta la concepción de ciencia, de aprendizaje y de enseñanza tanto desde la consideración del estudiante como también del docente. A partir de esto, es posible reconocer distintas posturas epistemológicas relacionadas con el desempeño áulico.

Retomando estas clasificaciones y trabajando sobre ellas, Guirado $(2013,2016)$ realiza una síntesis teórica al unificar en tres categorías los principales modelos didácticos. A continuación, en la Tabla 3, se presentan los aspectos más destacados de dicha síntesis, señalando para cada modelo la concepción de: ciencia, aprendizaje y enseñanza, como así también las características de los estudiantes, los docentes y las actividades experimentales:

TABLA 3. Modelos didácticos para la Enseñanza de las Ciencias

\begin{tabular}{|c|c|c|c|}
\hline & Modelo tradicional & Modelo por descubrimiento & Modelo constructivista \\
\hline Ciencia & $\begin{array}{l}\text { Es el estudio directo de la } \\
\text { realidad y se presenta como } \\
\text { un cuerpo cerrado de } \\
\text { conocimientos }\end{array}$ & $\begin{array}{l}\text { Se la concibe como la } \\
\text { generación de } \\
\text { conocimiento científico por } \\
\text { descubrimiento, estando } \\
\text { caracterizada por un } \\
\text { método particular }\end{array}$ & $\begin{array}{l}\text { Es una construcción histórico-- } \\
\text { social sobre modelos interpretativos } \\
\text { de la realidad, que parte del planteo } \\
\text { de problemas y búsqueda de } \\
\text { soluciones }\end{array}$ \\
\hline Aprendizaje & Memorístico y por recepción & Por descubrimiento & $\begin{array}{l}\text { Significativo, a través de la } \\
\text { construcción del conocimiento }\end{array}$ \\
\hline Enseñanza & Transmisiva & Por descubrimiento & $\begin{array}{l}\text { Mediante conflicto cognitivo y/o } \\
\text { por investigación dirigida } \\
\text { (Investigación en la escuela) }\end{array}$ \\
\hline Docente & $\begin{array}{l}\text { Es el transmisor de los } \\
\text { contenidos, es el que posee } \\
\text { los conocimientos y sus } \\
\text { explicaciones propician el } \\
\text { aprendizaje de los alumnos }\end{array}$ & $\begin{array}{l}\text { Es un coordinador de } \\
\text { actividades experimentales, } \\
\text { es quién debe propiciar } \\
\text { situaciones experimentales } \\
\text { que ayuden al estudiante a } \\
\text { desarrollar habilidades de } \\
\text { investigación. }\end{array}$ & $\begin{array}{l}\text { El profesor es guía de las } \\
\text { investigaciones de los alumnos con } \\
\text { adaptaciones constantes de las } \\
\text { actividades de aprendizaje }\end{array}$ \\
\hline Estudiantes & Alumno pasivo & $\begin{array}{l}\text { El alumno tiene un papel } \\
\text { importante y participativo } \\
\text { del alumno que realiza } \\
\text { diversas actividades de } \\
\text { carácter abierto y flexible y } \\
\text { frecuentemente en grupos }\end{array}$ & $\begin{array}{l}\text { Alumno constructor activo de su } \\
\text { propio conocimiento y } \\
\text { reconstructor de los contenidos } \\
\text { escolares a los que se enfrenta }\end{array}$ \\
\hline $\begin{array}{l}\text { Actividades } \\
\text { experimentales }\end{array}$ & $\begin{array}{l}\text { El laboratorio constituye una } \\
\text { instancia para demostrar la } \\
\text { teoría La planificación está a } \\
\text { cargo del docente, se basa en } \\
\text { objetivos definidos, en los } \\
\text { contenidos y en actividades } \\
\text { de fijación y comprobación }\end{array}$ & $\begin{array}{l}\text { Las actividades deben } \\
\text { propiciar situaciones } \\
\text { experimentales que ayuden } \\
\text { al estudiante a desarrollar } \\
\text { habilidades de } \\
\text { investigación }\end{array}$ & $\begin{array}{l}\text { Laboratorio de indagación e } \\
\text { investigación. Las actividades } \\
\text { experimentales deben partir de las } \\
\text { ideas previas de los alumnos, } \\
\text { otorgando importancia al contexto } \\
\text { en el que se presenta el } \\
\text { conocimiento científico. Es } \\
\text { importante el trabajo en pequeños } \\
\text { grupos y la interacción entre pares }\end{array}$ \\
\hline
\end{tabular}

Fuente: Adaptación de Guirado, 2013 
Avanzando en este concepto, Pontes Pedrajas, Poyato López y Oliva Martínez (2015), señalan que, al pensar en modelos didácticos, de manera general, se puede señalar la existencia de dos enfoques bien diferenciados y un enfoque intermedio. Así, coincidiendo con la clasificación de Guirado (2013), señalan la presencia de un enfoque tradicional o transmisivo, para el que predomina una visión del aprendizaje centrada en la transmisión de conocimientos y en el papel central que desempeña el profesor. En el otro extremo ubican un enfoque constructivista o innovador, con una visión del aprendizaje centrado en el alumno, que fomenta la construcción y evolución de su conocimiento. Entre ambos enfoques, proponen un enfoque intermedio que se caracteriza por ideas mixtas, similares a las descriptas por Guirado (2013) para el modelo por descubrimiento. Hernández y Maquilón (2010), destacan que tales ideas intermedias, al menos en lo que respecta a los procesos de aprendizaje, tienen más bien un carácter ambivalente entre el modelo educativo centrado en el alumno y el modelo centrado en el profesor.

\section{LOS MODELOS DIDÁCTICOS Y LOS TRABAJOS PRÁCTICOS DE LABORATORIO}

A partir de lo presentado en los apartados anteriores, se pueden inferir algunas vinculaciones. De esta manera, podría pensarse que tanto las actividades experimentales propuestas, como la forma de desarrollarlas, estarán basadas en el modelo didáctico que posee el docente y pone de manifiesto en el aula. Por esto, nos proponemos integrar las clasificaciones de modelos didácticos y TPL, lo cual podría contribuir con el análisis del trabajo experimental en el aula.

Así, las clasificaciones ya presentadas (Herron, 1971; Priestley, 1997; Guirado, 2013; Pontes Pedrajas, Poyato López y Oliva Martínez, 2015) se pueden vincular de la siguiente manera:

- El modelo didáctico tradicional presenta a la observación y la experimentación como los caminos válidos para conocer la realidad y el laboratorio constituye una instancia para demostrar la teoría. De esta forma la enseñanza de las ciencias es la transmisión de contenidos científicos gracias al docente, quien es el poseedor de dichos conocimientos. Esto nos permite inferir que los niveles de apertura a los que subyace este modelo son los niveles 1,2 y 3, según la clasificación esbozada por Priestley, ya que los procesos cognitivos requeridos en los estudiantes son la observación y la comprensión del conocimiento previamente elaborado por el docente. Así mismo, esta situación se vería reflejada en los niveles 0 y 1 de Herron, ya que pertenecen a prácticas expositivas, ya sean demostrativas o ejercicios realizados por los estudiantes donde objetivos, materiales y métodos están estipulados por el docente de manera previa.

- El modelo intermedio o mixto caracteriza a las ciencias por su método, considerando que es en la realidad externa donde se puede observar y encontrar el conocimiento. Por estas razones, la enseñanza de las ciencias se relaciona íntimamente con la coordinación de actividades experimentales centradas en procedimientos científicos, mientras que el aprendizaje se basa en el conocimiento de los procedimientos propios de las ciencias, para a partir de ellos descubrir los conocimientos. Así, las prácticas por investigación señaladas por Herron, correspondientes al nivel 2, se vincularían con este modelo. De manera similar, podemos encontrar esta correspondencia en la clasificación de Priestley, para los niveles de apertura 3, 4 y 5, donde los procesos cognitivos requeridos, además de la comprensión y apropiación de los contenidos, incluyen la aplicación de los mismos, teniendo en cuenta que para cada uno de estos niveles hay diferentes grados en la participación del docente en la realización de las prácticas.

- Por último, del modelo constructivista, considera a la ciencia como una construcción de modelos interpretativos de la realidad y a su enseñanza como el planteo de situaciones que permiten a los alumnos reelaborar contenidos, con el docente como guía de sus investigaciones. Es por esto que las investigaciones abiertas (nivel 3) y los proyectos (nivel 4), según Herron, se sustentarían en este modelo, ya que presentan actividades donde hasta los objetivos de las mismas pueden ser abiertos en parte, privilegiando la postura de un alumno constructor de su propio conocimiento y reconstructor de los contenidos escolares a los que debe enfrentarse. Además, los niveles de apertura 6 y 7 , 
esbozados por Priestley, se corresponderían con este modelo, debido a que los procesos cognitivos requeridos, además de la comprensión, la apropiación y la aplicación de los contenidos, incluye el análisis, síntesis y aplicación de los mismos.

A continuación, en las Tablas 4 y 5, se resume la relación entre los diferentes niveles de apertura de los TPL y la clasificación propuesta para modelos didácticos:

TABLA 4. Relación entre los niveles de apertura de los TPL según Herron (1971) y los modelos didácticos según Guirado (2013, 2016) y Pontes Pedrajas, Poyato López y Oliva Martínez (2015)

Niveles de apertura según Herron Modelo didáctico al que referencia

\begin{tabular}{ll}
\hline 1 & Tradicional \\
\hline 2 & Tradicional \\
\hline 3 & Intermedio \\
\hline 4 & Constructivista \\
\hline
\end{tabular}

Fuente: Elaboración propia

TABLA 5. Relación entre los niveles de apertura de los TPL según Priestley (1997) y los modelos didácticos según Guirado $(2013,2016)$ y Pontes Pedrajas, Poyato López y Oliva Martínez (2015)

\begin{tabular}{cc}
\hline Niveles de apertura según Priestley & Modelo didáctico al que referencia \\
\hline 1 & Tradicional \\
\hline 2 & Tradicional \\
\hline 3 & Intermedio \\
\hline 4 & Intermedio \\
\hline 5 & Intermedio \\
\hline 6 & Constructivista \\
\hline 7 & Constructivista
\end{tabular}

Fuente: Elaboración propia

\section{INTEGRACIÓN DE CLASIFICACIONES: UN APORTE METODOLÓGICO AL ANÁLISIS DE LOS TRABAJOS PRÁCTICOS DE LABORATORIO}

A continuación, se presenta una clasificación que se ha elaborado teniendo en cuenta las anteriores (Herron,1971; Priestley, 1997; Guirado, 2013 y Pontes Pedrajas, Poyato López y Oliva Martínez, 2015), en la que se asocia a cada nivel de apertura una descripción sintética de la propuesta de actividades, los procesos cognitivos requeridos por parte de los estudiantes, la presencia explícita o la ausencia en el protocolo de los objetivos, materiales, métodos y soluciones de dicha práctica, así como también quiénes son los sujetos que la realizan y el modelo didáctico que la sustenta. En la Tabla 6, se presenta el resumen de dicha clasificación: 
TABLA 6. Clasificación de los Trabajos Prácticos de Laboratorio.

\begin{tabular}{|c|c|c|c|c|c|c|c|c|c|}
\hline Nivel & Nombre & $\begin{array}{c}\text { Descripción de las actividades en el } \\
\text { laboratorio }\end{array}$ & $\begin{array}{l}\text { Procesos } \\
\text { cognitivos } \\
\text { requeridos }\end{array}$ & Objetivo & Material & Método & Solución & $\begin{array}{l}\text { Realización } \\
\text { de la práctica }\end{array}$ & Modelo didáctico \\
\hline 0 & Sin participación & $\begin{array}{c}\text { Sin protocolo o guía de trabajo. } \\
\text { Demostración por parte del profesor de un } \\
\text { fenómeno de interés o para demostración de } \\
\text { la teoría. Los estudiantes registran sus } \\
\text { observaciones no necesariamente en un } \\
\text { informe de laboratorio }\end{array}$ & Observación & Dado & Dado & Dado & Dad. & Docente & Tradicional \\
\hline 1 & $\begin{array}{l}\text { Herméticamente } \\
\text { cerrado }\end{array}$ & $\begin{array}{l}\text { En el protocolo se pautan todos los } \\
\text { procedimientos a los estudiantes, los cuales } \\
\text { anotan las observaciones en casilleros } \\
\text { reservados para tal fin en un informe de } \\
\text { laboratorio. Se incluyen tablas con datos }\end{array}$ & Conocimiento. & Dad & Dado & Dado & Dada & $\begin{array}{l}\text { Docente- } \\
\text { Alumnos }\end{array}$ & Tradicional \\
\hline 2 & Muy cerrado & $\begin{array}{c}\text { En el protocolo se pautan todos los } \\
\text { procedimientos a los estudiantes. Se } \\
\text { incluyen tablas de datos }\end{array}$ & Conocimiento & Dado & Dado & Dado & $\begin{array}{l}\text { Dada en } \\
\text { parte o } \\
\text { abierta }\end{array}$ & $\begin{array}{l}\text { Docente- } \\
\text { Alumnos }\end{array}$ & Tradicional \\
\hline 3 & Cerrado & $\begin{array}{l}\text { En el protocolo se pautan todos los } \\
\text { procedimientos a los estudiantes }\end{array}$ & $\begin{array}{l}\text { Conocimiento y } \\
\text { comprensión }\end{array}$ & Dado & Dado & Dado & Abierta & $\begin{array}{l}\text { Docente- } \\
\text { Alumnos }\end{array}$ & $\begin{array}{c}\text { Transición entre el } \\
\text { modelo tradicional y } \\
\text { el modelo intermedio }\end{array}$ \\
\hline 4 & Entreabierto & $\begin{array}{l}\text { En el protocolo se pautan todos los } \\
\text { procedimientos a los estudiantes. Algunas } \\
\text { preguntas o conclusiones son abiertas }\end{array}$ & $\begin{array}{l}\text { Conocimiento, } \\
\text { comprensión y } \\
\text { aplicación }\end{array}$ & Dado & $\begin{array}{l}\text { Dado } \\
\text { todo o en } \\
\text { parte }\end{array}$ & Dado & Abierta & $\begin{array}{l}\text { Alumnos- } \\
\text { Docente }\end{array}$ & Intermedio \\
\hline 5 & $\begin{array}{l}\text { Ligeramente } \\
\text { abierto }\end{array}$ & $\begin{array}{c}\text { En el protocolo se pautan la mayoría de los } \\
\text { procedimientos a los estudiantes y se } \\
\text { plantean algunas preguntas o cuestiones } \\
\text { abiertas }\end{array}$ & $\begin{array}{l}\text { Conocimiento, } \\
\text { comprensión y } \\
\text { aplicación }\end{array}$ & Dado & $\begin{array}{l}\text { Dado } \\
\text { todo o en } \\
\text { parte }\end{array}$ & $\begin{array}{l}\text { Dado } \\
\text { todo o en } \\
\text { parte }\end{array}$ & Abierta & $\begin{array}{l}\text { Alumnos- } \\
\text { Docente }\end{array}$ & $\begin{array}{l}\text { Transición entre el } \\
\text { modelo intermedio y } \\
\text { el constructivista }\end{array}$ \\
\hline 6 & Abierto & $\begin{array}{l}\text { Los estudiantes proponen sus propios } \\
\text { procedimientos. Se les proporciona una } \\
\text { lista con el material. Muchas de las } \\
\text { preguntas que se plantean son abiertas }\end{array}$ & $\begin{array}{l}\text { Conocimiento, } \\
\text { comprensión, } \\
\text { aplicación, } \\
\text { análisis y } \\
\text { síntesis }\end{array}$ & Dado & Abierto & Abierto & Abierta & Alumnos & Constructivista \\
\hline 7 & Muy abierto & $\begin{array}{l}\text { Se les plantea a los estudiantes un problema } \\
\text { que tienen que resolver (o ellos pueden } \\
\text { plantear el problema). El procedimiento es } \\
\text { propuesto por los estudiantes }\end{array}$ & $\begin{array}{l}\text { Conocimiento, } \\
\text { comprensión y } \\
\text { aplicación. } \\
\text { Análisis, } \\
\text { síntesis y } \\
\text { evaluación }\end{array}$ & $\begin{array}{l}\text { Dado en } \\
\text { parte o } \\
\text { abierto }\end{array}$ & Abierto & Abierto & Abierta & Alumnos & Constructivista \\
\hline
\end{tabular}

Fuente: Elaboración propia 
Retomando la relación entre los niveles de apertura de las prácticas experimentales y los modelos didácticos que las sustentan, es que puede inferirse que la apertura en las prácticas de laboratorio se encuentra asociada a un modelo didáctico particular. Por tal motivo se considera necesario analizar las clases de laboratorio, teniendo en cuenta los niveles de apertura y los modelos didácticos que las sustentan. A continuación, se analiza la propuesta presentada en la Tabla 6 .

- El nivel de apertura 0 de los TPL incluye demostraciones experimentales realizadas por el docente, para las que tanto los objetivos de la práctica como sus métodos y soluciones son conocidas con anterioridad, por lo tanto, requieren únicamente la observación de los estudiantes. Este tipo de prácticas experimentales podrían asociarse al modelo tradicional, ya que estas experiencias estarían cumpliendo la función de apoyo al discurso del docente, donde la función de los estudiantes es la apropiación formal de los conocimientos, a través de un proceso de captación, atención, retención y fijación de su contenido.

- En cuanto al nivel 1 de apertura, puede observarse una iniciación en la participación de los estudiantes en las prácticas experimentales, aunque al igual que en el nivel anterior, los objetivos de la práctica, métodos y soluciones son conocidas de antemano. Las prácticas presentan un alto grado de estructuración, con gran cantidad de detalles, y los procesos cognitivos que se fomentan en los estudiantes son únicamente la observación y la aplicación de conocimientos. Por lo expuesto, las actividades propuestas en este nivel, al igual que las de nivel 0 , aún se encontrarían asociadas con el modelo de enseñanza tradicional.

- Las actividades de laboratorio muy cerradas (nivel 2 de apertura), sólo se diferencian con las de nivel 1 en que la solución a las mismas puede ser dada en parte o no estar dadas. Los procesos cognitivos que se favorecen son la observación y la aplicación de conocimientos, y los objetivos, materiales y métodos están detallados previamente por el docente. Por esta misma razón, al igual que las prácticas de nivel 0 y 1 , los TPL de este nivel aún se encontrarían sustentados en el modelo tradicional.

- En las prácticas experimentales cerradas, con nivel 3 de apertura, las soluciones a las mismas son abiertas, y aunque todos los procedimientos sean facilitados a los estudiantes, los procesos cognitivos involucrados son, además de la observación y la aplicación del conocimiento, la comprensión. El hecho de que las experiencias continúen siendo dirigidas por el docente, aunque presenten relación con el entorno, de manera de hacer las prácticas más atractivas, situaría a este tipo de TPL a modo de transición entre el modelo tradicional y el modelo didáctico intermedio.

- En los TPL con nivel de apertura 4, se suma a los procesos cognitivos de la observación, el conocimiento y la comprensión, la aplicación de dicho conocimiento. A partir de este nivel, el docente disminuye su participación en la práctica y aumenta la toma de decisiones de los estudiantes al tener que determinar, por ejemplo, qué materiales usar para alcanzar los objetivos propuestos. Esta situación se correspondería como en el caso anterior, con el modelo didáctico intermedio, ya que los alumnos se encuentran involucrados en la planificación y realización de la práctica, con el docente como coordinador de las prácticas.

- El nivel de apertura 5 considera las mismas características que el anterior, pero aumentando el grado de participación de los estudiantes, no sólo en la selección de materiales, sino también en la de los métodos adecuados para llevar a cabo la práctica. Los estudiantes asumen una posición aún más activa, planteando su postura frente a la información que se está abordando. De esta manera podría considerarse que este nivel de apertura ubicado en una transición entre modelos, ya que presenta rasgos tanto del modelo intermedio como del modelo constructivista.

- Avanzando en la escala de niveles de apertura, puede observarse que en el nivel 6 disminuye drásticamente la participación del docente. Son los estudiantes los que proponen los métodos y solamente son propuestos por el docente los objetivos de la práctica. De esta manera, los procesos cognitivos requeridos incluyen el análisis y la síntesis. En este tipo de TPL se plantean problemas de 
interés para los estudiantes, ya que son ellos quienes eligen el diseño y las situaciones a resolver. Así, los TPL ubicados en este nivel se corresponderían con el modelo didáctico constructivista.

- El último nivel de apertura, al igual que el anterior, se vincula con el modelo didáctico constructivista, debido a que se plantean situaciones problemáticas con problemas abiertos que no tienen una solución inmediata y se presentan situaciones donde los estudiantes a través de la experimentación deben obtener respuestas a los interrogantes planteados.

\section{REFLEXIONES FINALES}

La clasificación y caracterización de los TPL que se realizan para la enseñanza de las Ciencias Naturales resulta una cuestión esencial que contribuye con el quehacer docente, ya que permite visualizar de manera esquemática las características del TPL realizado y los objetivos que pueden alcanzarse. Por otra parte, atendiendo a que en múltiples investigaciones vinculadas a la enseñanza y al aprendizaje de las Ciencias Naturales se señala la importancia de reflexionar sobre los TPL propuestos tanto para el nivel secundario como universitario (Cano, 2019; Zorrilla, Morales, Mazzitelli y Olivera, 2019), una clasificación permite también, buscar nuevas aproximaciones hacia dónde se quiere dirigir el trabajo experimental, en función de diferentes variables.

Cabe destacar que no existe una manera objetiva de determinar la "calidad" de una clasificación, ya que una taxonomía puede ser muy útil para un contexto, pero no así para otros. De esta manera, cualquier clasificación que se presente será factible de modificaciones, en función de los intereses del docente o del estudiante que se enfrente a ella.

En este punto, hay que señalar que para modificar el tipo de TPL que muchos docentes realizan en su enseñanza habitual, es necesario ir más allá de solamente mostrar que deben modificar las características de las actividades que proponen, ya que, si no se explicitan claramente los supuestos de base, reflexionando sobre su alcance tanto en la enseñanza como en el aprendizaje, y no se plantea la necesidad de modificar también dichos supuestos, entonces no se estará favoreciendo un cambio de largo alcance. En tal sentido, la clasificación propuesta en este artículo pretender ser una herramienta metodológica útil para avanzar en ese camino.

\section{Referencias}

Antúnez, G. C., Pérez, S. M. y Petrucci, D. (2008). Concepciones de los docentes universitarios sobre los trabajos prácticos de laboratorio. Revista Brasileira de Pesquisa em Educação em Ciências, $8(1), 1-17$. Recuperado de https://periodicos.ufmg.br/index.php/rbpec/article/view/4028 [28 de julio de 2020].

Barberá, O. y Valdés, P. (1996). El trabajo práctico en la enseñanza de las ciencias: una revisión. Enseñanza de las ciencias: revista de investigación y experiencias didácticas, 14(3), 365-379. Recuperado de https://www.raco.cat/index.php/Ensenanza/article/view/21466 [28 de julio de 2020].

Bastida de la Calle, M., Ramos Fernández, F. y Soto López, J. (1990). Prácticas de laboratorio:¿una inversión poco rentable? Revista Investigación en la Escuela, 11, 77-91. Recuperado de https://idus.us.es/bitstream/handle/11441/59263/Pr\%c3\%alcticas\%20de\%20laboratorio\%20u na $\% 20$ inversi $\%$ c3\%b3n\%20poco $\% 20$ rentable.pdf?sequence $=1 \&$ isAllowed $=\mathrm{y}$ [ 28 de julio de 2020].

Bravo, A. A., Ramírez, G. P., Faúndez, C. A. y Astudillo, H. F. (2016). Propuesta didáctica constructivista para la adquisición de aprendizajes significativos de conceptos en Física de fluidos. Formación universitaria, 9(2), 105-114. DOI: $10.4067 / \mathrm{S} 0718-50062016000200012$

Caamaño, A. (1992). Los trabajos prácticos en ciencias experimentales. Una reflexión sobre sus objetivos y una propuesta para su diversificación. Aula de Innovación Educativa, 9, 61-68. 
Cano, M. C. (2019). ABP: repensando los laboratorios de química. REDU: Revista de Docencia Universitaria, 17(2), 25-39. DOI: 10.4995/redu.2019.11667

Carrascosa, J.; Vilches, A. y Valdés, P. (2006). Papel de la actividad experimental en la educación científica. Caderno Brasileiro de Ensino de Física, 23(2), 157-181. Recuperado de https://dialnet.unirioja.es/servlet/articulo?codigo=5166022 [28 de julio de 2020].

Chinchilla Buelvas, F. J. (2017). Enseñanza de la física orientando la práctica experimental como investigación. Revista Científica, Zulia, 2, 181-194. Recuperado de https://go.gale.com/ps/anonymous?id=GALE\%7CA500224014\&sid=googleScholar\&v=2.1\& $\mathrm{it}=\mathrm{r} \&$ linkaccess $=\mathrm{abs} \& \mathrm{issn}=01242253 \& \mathrm{p}=\mathrm{IFME \& sw}=\mathrm{w}$ [28 de julio de 2020].

Chrobak, R. y Leiva Benegas, M. (2006). Mapas conceptuales y modelos didácticos de profesores de química. Concept Maps: Theory, Methodology, Technology. Proc. of the Second Int. Conference on Concept Mapping. Universidad de Costa Rica, San José, Costa Rica, 415-422.

Cruz, M. D. T., Sanz, J. A. P. y León, M. R. (2019). La aplicación del enfoque investigativo integrador en las prácticas de laboratorio para la formación de profesores de Química. Pedagogía Universitaria, $24(2), \quad 53 . \quad$ Recuperado https://go.gale.com/ps/anonymous?id=GALE\%7CA613923653\&sid=googleScholar\&v=2.1\&

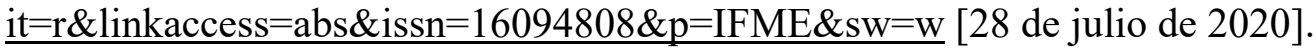

De Pro Bueno, A. (1998). ¿Se pueden enseñar contenidos procedimentales en las clases de ciencias? Enseñanza de las ciencias: revista de investigación y experiencias didácticas, 16(1), 21-41. Recuperado de https://www.raco.cat/index.php/Ensenanza/article/view/83200 [28 de julio de 2020].

De Pro Bueno, A. (1999). Planificación de unidades didácticas por los profesores: análisis de tipos de actividades de enseñanza. Enseñanza de las ciencias: revista de investigación y experiencias didácticas, $\quad 17(3), \quad 411-429 . \quad$ Recuperado de https://www.raco.cat/index.php/Ensenanza/article/download/21594/21428/0 [28 de julio de 2020].

Del Carmen, L. (2000).Los trabajos prácticos. En: F. Perales Palacios y P. Cañal de León (coord). Didáctica de las ciencias experimentales. Teoría y práctica de la Enseñanza de las Ciencias (267-288). España: Marfil, S. A.

Del Carmen, L. (2011). El lugar de los trabajos prácticos en la construcción del conocimiento científico en la enseñanza de la biología y la geología. En A. Caamaño (coord.). Didáctica de la biología y la geología. Formación del profesorado. Educación secundaria (91-108). Barcelona: Grao.

Fernández, J.; Elortegui, N.; Rodríguez, J.F. y Moreno, T. (1997). ¿Qué idea se tiene de la ciencia desde los modelos didácticos? Alambique, 12, 87-99. Recuperado de http://www.grupoblascabrera.org/webs/ficheros/08\%20Bibliograf\%C3\%ADa/03\%20Moddid/ 22\%20Idea\%20ciencia\%20modelos\%20didacticos.pdf [28 de julio de 2020].

Fernández, J. y Elortegui, N. (1996). Qué piensan los profesores de cómo se debe enseñar. Enseñanza de las ciencias: revista de investigación y experiencias didácticas, 14(3), 331-342. Recuperado de https://www.raco.cat/index.php/Ensenanza/article/view/56871 [28 de julio de 2020].

Fernández, N. (coord). (2010). Algo más que locos experimentos para hacer en clases. Manual de trabajos de laboratorio. Argentina: Editorial Utopias.

Fernández, N. E. (2013). Los Trabajos Prácticos de Laboratorio por investigación en la enseñanza de la Biología. Revista de Educación en Biología, 16(2), 15-30. Recuperado de https://revistas.psi.unc.edu.ar/index.php/revistaadbia/article/view/22395/22013 [28 de julio de 2020].

Fernández, J.; Elortegui, N. y Medina, M. (2002). Consideraciones sobre la investigación en didáctica de las Ciencias de la Naturaleza. Revista Alambique, 34, 37-46.

Flores, J., Caballero Sahelices, M. C. y Moreira, M. A. (2009). El laboratorio en la enseñanza de las ciencias: Una visión integral en este complejo ambiente de aprendizaje. Revista de 
investigación,

$33(68)$,

75-111.

Recuperado

de https://www.redalyc.org/pdf/3761/376140383004.pdf [28 de julio de 2020].

García Ruíz, M. y Calixto Flores, R. (1999). Actividades experimentales para la enseñanza de las ciencias naturales en educación básica. Perfiles educativos, 84. Recuperado de https://www.redalyc.org/pdf/132/13208408.pdf [28 de julio de 2020].

Gil, S. (1997). Nuevas tecnologías en la enseñanza de la física oportunidades y desafíos. En: Memorias VI Conferencia Interamericana sobre Educación en la Física, 13-15.

Guirado, A. M. (2013). Los Modelos Didácticos de docentes de Ciencias Naturales de nivel secundario: reconstrucción a partir de sus concepciones y sus prácticas áulicas. Tesis de doctorado. Universidad Nacional de Cuyo, Mendoza, Argentina.

Guirado, A. M. (2016). Los modelos didácticos de docentes de Ciencias Naturales de nivel secundario: reconstrucción a partir de sus concepciones y sus prácticas áulicas. Revista de Enseñanza de la Física, 28(2), 111-112. Recuperado de https://revistas.unc.edu.ar/index.php/revistaEF/article/view/15819/15628 [21 de diciembre de 2020].

Hernández, F. y Maquilón, J. (2010). Las concepciones de la enseñanza. Aportaciones para la formación del profesorado. Revista Electrónica Interuniversitaria de Formación del Profesorado, 13(3), 17-25. $\quad$ Recuperado de https://dialnet.unirioja.es/servlet/articulo?codigo $=3307370$ [28 de julio de 2020].

Herron, M. D. (1971). The nature of scientific enquiry. The School Review, 79(2), 171-212.

Hodson, D. (1994). Hacia un enfoque más crítico del trabajo de laboratorio. Enseñanza de las ciencias, 12(3), 299-313.

Idoyaga, I. y Maeyoshimoto, J. (2018). Las actividades experimentales simples: una alternativa para la enseñanza de la física. En M.G. Lorenzo; H.S. Odetti y A.E. Ortolani (Ed.). Comunicando la ciencia (55-67). Argentina: Ediciones UNL.

Insausti, M. J., y Merino, M. (2016). Una propuesta para el aprendizaje de contenidos procedimentales en el laboratorio de física y química. Investigações em Ensino de Ciências, 5(2), 93-119. Recuperado de https://www.if.ufrgs.br/cref/ojs/index.php/ienci/article/view/613/402 [28 de julio de 2020].

Jiménez Llanos, A. B. y Correa Piñero, A. D. (2002). El modelo de teorías implícitas en el análisis de la estructura de creencias del profesorado universitario sobre la enseñanza. Revista de Investigación Educativa, 20(2), 525-548. Recuperado de https://revistas.um.es/rie/article/view/99051 [28 de julio de 2020].

Jiménez Valverde, G., Llobera Jiménez, R. y Llitjós Viza, A. (2006). La atención a la diversidad en las prácticas de laboratorio de química: los niveles de abertura. Enseñanza de las ciencias: revista de investigación y experiencias didácticas, 24(1), 59-70. Recuperado de https://www.raco.cat/index.php/Ensenanza/article/view/73532 [28 de julio de 2020].

Leite, L. y Figueiroa, A. (2004). Las actividades de laboratorio y la explicación científica en los manuales escolares de ciencias. Alambique. Didáctica de las Ciencias Experimentales, 39, 2030 .

López Rua, A. M., y Tamayo Alzate, Ó. E. (2012). Las prácticas de laboratorio en la enseñanza de las ciencias naturales. Revista Latinoamericana de Estudios Educativos, 8(1), 145-166. Recuperado de https://www.redalyc.org/pdf/1341/134129256008.pdf [28 de julio de 2020].

Martínez Aznar, M., Martín del Pozo, R., Rodrigo Vega, M., Varela Nieto, M., Fernández Lozano, M. y Guerrero Serón, A. (2001). ¿Qué pensamiento profesional y curricular tienen los futuros profesores de ciencias de secundaria? Enseñanza de las ciencias, 19(1), 67-87. Recuperado de https://ddd.uab.cat/record/1523 [28 de julio de 2020].

Merino, J. M. y Herrero F. (2007). Resolución de problemas experimentales de Química: una alternativa a las prácticas tradicionales. Revista Electrónica de Enseñanza de las Ciencias, 6(3), 
630-648. Recuperado de https://reec.uvigo.es/volumenes/volumen6/ART9 Vol6 N3.pdf [28 de julio de 2020].

Millar, R. (2009). Analysing practical activities to assess and improve effectiveness: The Practical Activity Analysis Inventory (PAAI). York: Centre for Innovation and Research in Science Education, University of York.

Nappa, N., Vázquez, S., Maratta, A. y Mazzitelli, C. (2015). Protocolos innovadores de prácticos de Química. Actas de la XVI Reunión de Educadores en la Química, 397-400

Pavón, Z. S., Soto, J. M. B., Prieto, C. A., y Araque, J. A. (2009). Las prácticas de laboratorio en la formación del profesorado de química. Un primer acercamiento. Diálogos educativos, 18, 9. Recuperado https://www.researchgate.net/profile/Zenahir_Siso2/publication/41952646 Las practicas de laboratorio_en la formacion_del profesorado de Quimica_Un_primer_acercamiento/links/5 7f649af08ae886b8981b889/Las-practicas-de-laboratorio-en-la-formacion-del-profesorado-deQuimica-Un-primer-acercamiento.pdf [28 de julio de 2020].

Pontes Pedrajas, A., Poyato López, F. J., y Oliva Martínez, J. M. (2015). Concepciones sobre el aprendizaje en estudiantes del Máster de profesorado de Educación Secundaria del área de ciencia y tecnología. Profesorado. Revista de Currículum y Formación de Profesorado, 19(2), 225-243. Recuperado de https://www.redalyc.org/pdf/567/56741181015.pdf [28 de julio de 2020].

Petrucci D., Ure J. y Salomone, H. D. (2011). Cómo ven a los trabajos prácticos de laboratorio de física los estudiantes universitarios. Revista de Enseñanza de la Física, 19(1), 7-19. Recuperado de https://revistas.psi.unc.edu.ar/index.php/revistaEF/article/view/8060/8915 [28 de julio de 2020].

Porlán, A., Rivero, A., y Martín, R. (1997). Conocimiento profesional y epistemología de los profesores I: Teoría, métodos e instrumentos. Enseñanza de las ciencias: revista de investigación y experiencias didácticas, 15(2), 155-171.

Porlán, R., Rivero, A., y Martín, R. (1998). Conocimiento profesional y epistemología de los profesores, II: Estudios empíricos y conclusiones. Enseñanza de las Ciencias, 16(2), 271-288.

Priestley, W. (1997). The impact of longer term intervention on reforming physical science teachers' approaches to laboratory instruction: seeking a more effective role for laboratory in science education. Dissertation Abstracts International, 58(3), 806.

Richoux, H. y Beaufils, D. (2003).La planificación de las actividades de los estudiantes en los trabajos prácticos de física: análisis de prácticas de profesores. Enseñanza de las Ciencias, 21(1), 95106. Recuperado de https://ddd.uab.cat/record/1597 [28 de julio de 2020].

Rodríguez Garrido, E. A. y Meneses Villagrá, J. A., (2011). Las concepciones y creencias de profesores de ciencias naturales sobre ciencia, su enseñanza y aprendizaje, mediadas por la formación inicial, la educación continuada y la experiencia profesional. Revista Brasileira de Pesquisa em Educação em Ciências, 5(2). Recuperado de https://periodicos.ufmg.br/index.php/rbpec/article/view/4061 [28 de julio de 2020].

Ruiz Ortega, F. J. (2007). Modelos didácticos para la enseñanza de las Ciencias Naturales. Revista Latinoamericana de Estudios Educativos, 3(2), 41-60. Recuperado de https://www.redalyc.org/pdf/1341/134112600004.pdf [28 de julio de 2020].

Schwab, J. J. (1962). The teaching of science as enquiry. En J. J. Schwab y P. F. Brandwein (Eds.). The teaching of Science (pp. 3-103). Cambridge: Harvard University Press.

Tamir, P. y García Rovira, M. P. (1992). Características de los ejercicios de prácticas de laboratorio incluidos en los libros de textos de Ciencias utilizados en Cataluña. Enseñanza de las ciencias: revista de investigación y experiencias didácticas, 10(1), 3-12. Recuperado de https://ddd.uab.cat/record/23542 [28 de julio de 2020]. 
Valencia, K. y Torres, T. (2017). Impacto formativo de las prácticas de laboratorio en la formación de profesores de ciencias. Enseñanza de las ciencias, (Extra), 3033-3038. Recuperado de https://www.raco.cat/index.php/Ensenanza/article/view/336979 [28 de julio de 2020].

Valencia, K. (2017). Evaluación de las actividades de laboratorio en la formación de profesores de ciencias: un análisis crítico sobre su contribución a una visión contemporánea de la ciencia y el trabajo cientifico. Tesis de Maestría. Facultad de Educación. Universidad de Antioquía. Colombia.

Walz, M. V., Weisz, R. M. y Albarenque, R. L. (2013). El trabajo experimental en Física como estrategia de motivación. Un trabajo de años. Revista de la Escuela de Ciencias de la Educación, 8. DOI: 10.35305/rece.v0i8.161

Zorrilla, E. (2019). Las prácticas de laboratorio en la enseñanza y el aprendizaje de las Ciencias Naturales desde una perspectiva psicosocial. Tesis doctoral. Facultad de Filosofía y Letras, Universidad Nacional de Cuyo. Mendoza, Argentina.

Zorrilla, E. G., Morales, L., Mazzitelli, C. A. y Olivera, A. (2019). Análisis de trabajos prácticos de laboratorio elaborados por futuros docentes de Ciencias Naturales. Góndola, enseñanza y aprendizaje de las ciencias, 14(2), 286-302.DOI: 10.14483/23464712.13750

Zorrilla, E.G., Mazzitelli, C. A., Calle Restrepo, A., Angulo Delgado, F. y Soto Lombana, C. (2020). Las prácticas experimentales en el contexto de la formación docente inicial en Colombia. En Buteler, L. (coord). Actas del Décimo Quinto Simposio de Investigación en Educación en Física. 15. APFA, Argentina.

\section{CÓMO CITAR ESTE ARTÍCULO}

Zorrilla, E. G. y Mazzitelli, C. A. (2021). Trabajos Prácticos de Laboratorio y Modelos didácticos: una propuesta de clasificación. Didáctica de las ciencias experimentales y sociales, 40, 133-148. DOI: 10.7203/DCES.40.18056 
